\title{
The non-radial component of the solar wind and motion of dust near mean motion resonances with planets
}

\author{
J. Klačka, L. Kómar, P. Pástor, and J. Petržala
}

\begin{abstract}
Department of Astronomy, Physics of the Earth, and Meteorology, Faculty of Mathematics, Physics and Informatics, Comenius University, Mlynská dolina, 84248 Bratislava, Slovak Republic e-mail: [klacka;pavol.pastor]@fmph.uniba.sk
\end{abstract}

Received 28 September 2007 / Accepted 28 May 2008

\section{ABSTRACT}

\begin{abstract}
We investigate the effect of solar wind and solar electromagnetic radiation on the dynamics of spherical cosmic dust particles. We also consider the non-radial component of the solar wind velocity, in the reference frame of the Sun. We apply the equation of motion to the motion of dust grains near commensurability resonances with a planet - mean motion orbital resonance (MMR; a particle is in resonance with a planet when the ratio of their mean motions is approximately the ratio of two small integers) - and possible capture of the grains in the resonances. Up to now, only nonspherical grains, under action of the electromagnetic radiation of the central star, were known to exhibit an increase of semimajor axis before capture into the MMR. This paper shows that the same result can be generated by the non-radial component of the solar wind even for spherical dust particles. Spherical dust grains enable the treatment of the problem in an analytic way (at least partially), which is not the case for the effect of electromagnetic radiation on nonspherical dust grains. The situation treated in the paper presents the second known case when resonant trapping of a cosmic body occurs for diverging orbits. The paper presents the first case of secular evolution of the eccentricity of a body captured in the resonance derived in an analytic way for a body characterized by a diverging orbit.
\end{abstract}

Key words. scattering - celestial mechanics - interplanetary medium - solar wind

\section{Introduction}

Commensurability resonances have been well-known since the time of Kirkwood, who found gaps in the distribution of asteroidal semimajor axes (histogram: number of known asteroids versus their semimajor axes) in the asteroid belt in 1857. A body is in resonance with a planet when the ratio of their mean motions (mean motion $n=2 \pi / T$, where $T$ is orbital period) is the ratio of two small integers: the semimajor axis of the body oscillates around a constant value. The physics of these mean motion resonances with Solar System planets has been successfully studied since 1980 (Wisdom 1980). The orbital evolution of dust grains near resonances with planets has been intensively studied during the last twenty years (Jackson \& Zook 1989). Besides gravitational forces of the Sun and planet(s), the effect of solar electromagnetic radiation in the form of the Poynting-Robertson effect is also usually taken into account, including the effect of the solar wind: e.g., Jackson \& Zook (1989), Šidlichovský \& Nesvorný (1994), Beaugé \& Ferraz-Mello (1994), Marzari \& Vanzani (1994), Liou \& Zook (1995), Liou et al. (1995), Liou \& Zook (1997), Liou et al. (1999), Kehoe et al. (2002), Holmes et al. (2003). Observations confirming the existence of a dust ring around the Sun in resonance with the Earth are discussed in Brownlee (1994), Dermott et al. (1994), Reach et al. (1995). Qualitatively new results are obtained when we allow that real particles are nonspherical and instead of the Poynting-Robertson (P-R) effect (based on the assumption that material within dust grains is distributed in a spherically symmetric way), we use Eqs. (40), (41) of Klačka (2004) (plus gravitational acceleration of the third body, a planet) in modeling mean motion resonances. The generalization of the P-R effect considers interaction of the incident electromagnetic radiation with nonspherical dust grains and, thus, non-radial radiation pressure terms also are present in the general covariant equation of motion (Eq. (41) in Klačka 2004). For an application of the equation of motion to mean motion resonances, we refer to Klačka et al. (2005a,b) and Klačka \& Kocifaj (2006a,b).

The orbital evolution of spherical dust grains in the circular restricted three-body problem, with the simultaneous action of solar electromagnetic radiation (and a radial solar wind), is characterized by several important results. In the zone of mean motion orbital resonances with a planet the fundamental results are (see, e.g., Liou \& Zook 1997):

i) the secular evolution of the semimajor axis is a decreasing function of time near the resonances $(\langle\mathrm{d} a / \mathrm{d} t\rangle=0$ in the resonance);

ii) the secular evolution of the eccentricity in the exterior resonance is either an increasing function of time or a decreasing function of time and the values of eccentricity cannot reach a limiting value $e_{\mathrm{lim}}$, given by the condition $n_{\mathrm{P}} / n=$ $\left(1+3 e_{\lim }^{2} / 2\right) /\left(1-e_{\lim }^{2}\right)^{3 / 2} ; n_{\mathrm{P}}=2 \pi / T_{\mathrm{P}}$, where $T_{\mathrm{P}}$ is orbital period of the planet;

iii) the secular evolution of the eccentricity in the interior resonance is a decreasing function of time.

Resonant captures of arbitrarily shaped dust grains exist for exterior and interior mean motion resonances with planets (Klačka et al. 2005a,b; and Klačka \& Kocifaj 2006a,b). While capture of spherical grains in a resonance is characterized by the decrease of the semimajor axis and eccentricity outside the resonance, nonspherical grains may exhibit an increase of the semimajor axis (and eccentricity) outside the resonance (see the papers just 
mentioned). Resonant trapping is not normally expected for diverging orbits, such as the trapping found in detailed numerical integrations: this is the first case where a physically justified force can generate such a behavior.

The aim of our paper is to take into account the newest observational results on the solar wind. We are interested in the effect of the non-radial velocity component of the solar wind (in the reference frame of the Sun) on the motion of dust grains in the regions of the mean motion orbital resonances. Up to now, it was standardly used that the nonradial component of the solar wind velocity is zero (e.g., Grün et al. 1994; Liou \& Zook 1997) and that the azimuthal velocity component decreases as $1 / r$ with the heliocentric distance $r$ (e.g., Hundhausen 1972, p. 87; Stix 2002, pp. 424-425). According to these results, the non-radial velocity component of the solar wind should be $1 \mathrm{~km} \mathrm{~s}^{-1}$ at $1 \mathrm{AU}$, which is a very small value in comparison with the radial velocity component equal to $400 \mathrm{~km} \mathrm{~s}^{-1}$ for a low velocity solar wind (the value of $400 \mathrm{~km} \mathrm{~s}^{-1}$ is standardly used in the modeling of the orbital evolution of interplanetary dust, e.g., Dohnanyi 1978; Grün et al. 1994; Liou \& Zook 1997). However, the new observations of the solar wind show that the non-radial velocity component of the solar wind is not a decreasing function of heliocentric distance, at least for the distances covered by observations from Helios 2 during its first solar mission in 1976 (Bruno et al. 2003). The angle between the solar wind velocity vector and the radial heliocentric vector is close to $3^{\circ}$ for a low velocity solar wind. We will use the observational value in modeling the orbital evolution of dust grains near mean motion orbital resonances with the planet Neptune. We will discuss spherical dust grains, since they enable a partial analytical treatment. The solar wind and Poynting-Robertson effect will be considered, together with the circular restricted three-body problem.

Section 2 of the paper presents the fundamental definitions for the mean motion orbital resonances with planets and a formulation of the relevant equation of motion is given in Sect. 3. The secular orbital evolution of the dust particle under the action of the considered nongravitational forces is discussed in Sect. 4. The results of Sect. 4 are used in Sect. 5, which deals with the secular evolution of eccentricity in a mean motion resonance for the case of the circular restricted three-body problem. Section 5 presents both analytical and numerical results. The new results are presented in Sect. 5 and in Sect. 6, which shows that spherical dust grains can be captured in a mean motion orbital resonance even for the case when the grain's secular evolution of the semimajor axis is an increasing function of time before capture in the resonance. This behavior is due to the non-radial component of the solar wind.

\section{Mean-motion orbital resonances with planets}

A particle is in the $q$ th order exterior mean motion resonance with a planet if the ratio of its mean motion $n$ and the mean motion of the planet $n_{\mathrm{P}}$ approximately satisfy the equation $n_{\mathrm{P}} / n=$ $(p+q) / p$, where $p$ and $q$ are integer numbers. Similarly, the $q$ th order interior mean motion resonance is defined by the relation $n_{\mathrm{P}} / n=p /(p+q)$, where $p$ and $q$ are integer numbers. In terms of orbital periods: $T / T_{\mathrm{P}}=(p+q) / p$ for the exterior and $T / T_{\mathrm{P}}=p /(p+q)$ for the interior resonance.

We can determine the semimajor axis of the particle in the mean motion resonance from Kepler's third law. We have

$a^{3} n^{2}=G M_{\odot}(1-\beta)$,

$a_{\mathrm{P}}^{3} n_{\mathrm{P}}^{2}=G\left(M_{\odot}+m_{\mathrm{P}}\right)$, where $a, a_{\mathrm{P}}$ are semimajor axes of the particle characterized by the optical parameter $\beta$ and a planet with mass $m_{\mathrm{P}}$ orbiting the Sun of mass $M_{\odot}$. The first part of Eq. (1) uses the fact that central Keplerian acceleration is given by the sum of solar gravitational acceleration and radial component of radiation pressure acceleration. Equation (1) yields

$a=(1-\beta)^{1 / 3}\left(\frac{n_{\mathrm{P}}}{n}\right)^{2 / 3}\left(1+\frac{m_{\mathrm{P}}}{M_{\odot}}\right)^{-1 / 3} a_{\mathrm{P}}$.

On the basis of definitions of mean motion resonance and Eq. (2), we can write

$a=(1-\beta)^{1 / 3}\left(\frac{p+q}{\mathrm{p}}\right)^{2 / 3}\left(1+\frac{m_{\mathrm{P}}}{M_{\odot}}\right)^{-1 / 3} a_{\mathrm{P}}$

for the semimajor axis of the particle in the $q$ th order exterior resonance with a planet of mass $m_{\mathrm{P}}$. A similar relation can be obtained for the interior resonance.

\section{Equation of motion for a spherical dust particle}

Let us consider spherical dust grains under the action of gravitational forces generated by the Sun and a planet moving around the Sun. The grain is evolving under the action of solar electromagnetic radiation and the solar wind (we discuss micron sized and larger particles; numerical calculations are done for scales tens of microns in diameter, so we can neglect the action of the Lorentz force and mutual collisions - Holmes et al. 2003, state that the effect of the Lorentz force or the effect of neutral interstellar gas drag do not significantly change the results of simulations). The equation of motion of the particle is considered in the form

$$
\begin{aligned}
\frac{\mathrm{d} \boldsymbol{v}}{\mathrm{d} t}= & -\frac{\mu(1-\beta)}{r^{2}} \boldsymbol{e}_{\mathrm{R}} \\
& -\beta\left(1+\frac{\eta}{\bar{Q}_{\mathrm{pr}}^{\prime}}\right) \frac{\mu}{r^{2}}\left(\frac{\boldsymbol{v} \cdot \boldsymbol{e}_{\mathrm{R}}}{c} \boldsymbol{e}_{\mathrm{R}}+\frac{\boldsymbol{v}}{c}\right) \\
& +\beta \frac{\eta}{\bar{Q}_{\mathrm{pr}}^{\prime}} \frac{\mu}{r^{2}}\left\{\left(-\gamma_{\mathrm{T}} \frac{\boldsymbol{v} \cdot \boldsymbol{e}_{\mathrm{T}}}{c}+\frac{1}{2} \frac{v^{2}}{c u}\right) \boldsymbol{e}_{\mathrm{R}}\right. \\
& \left.+\gamma_{\mathrm{T}}\left(\frac{u}{c}-\frac{\boldsymbol{v} \cdot \boldsymbol{e}_{\mathrm{R}}}{c}\right) \boldsymbol{e}_{T}+\left(\frac{\boldsymbol{v} \cdot \boldsymbol{e}_{\mathrm{R}}}{c}\right) \frac{\boldsymbol{v}}{u}\right\} \\
& -\mu \frac{m_{\mathrm{P}}}{M_{\odot}}\left\{\frac{\boldsymbol{r}-\boldsymbol{r}_{\mathrm{P}}}{\left|\boldsymbol{r}-\boldsymbol{r}_{\mathrm{P}}\right|^{3}}+\frac{\boldsymbol{r}_{\mathrm{P}}}{\left|\boldsymbol{r}_{\mathrm{P}}\right|^{3}}\right\},
\end{aligned}
$$

where $\boldsymbol{v}=v_{\mathrm{R}} \boldsymbol{e}_{\mathrm{R}}+v_{\mathrm{T}} \boldsymbol{e}_{\mathrm{T}}, v=|\boldsymbol{v}|, r=|\boldsymbol{r}|, \boldsymbol{e}_{\mathrm{R}}=\boldsymbol{r} / r,\left|\boldsymbol{e}_{\mathrm{T}}\right|=1, \boldsymbol{e}_{\mathrm{T}} \cdot \boldsymbol{e}_{\mathrm{R}}=$ $0, r$ and $r_{\mathrm{P}}$ are position vectors of the particle and the planet (mass $m_{\mathrm{P}}$ ) with respect to the Sun (mass $M_{\odot}$ ), $\mu=G M_{\odot}$ and $G$ is the gravitational constant, $\beta=5.7 \times 10^{-5} \bar{Q}_{\mathrm{pr}}^{\prime} /\left(\varrho\left[\mathrm{g} / \mathrm{cm}^{3}\right] s[\mathrm{~cm}]\right)$, where $\varrho$ is the mass density of the particle and $s$ its radius, $\bar{Q}_{\mathrm{pr}}^{\prime}$ is the efficiency factor for radiation pressure (see, e.g., van de Hulst 1981) integrated over the solar spectrum and calculated for the radial direction. The positive values of $\bar{Q}_{\mathrm{pr}}^{\prime}$ depend on the optical properties of the particle, and $\bar{Q}_{\mathrm{pr}}^{\prime}<3$, approximately. For the Poynting-Robertson effect, we use the equation of motion derived by Robertson (1937) and, in a complete form, by Klačka (2000, 2004, 2008). The values $\eta=1 / 3$ and $u=400 \mathrm{~km} \mathrm{~s}^{-1}$ (the speed of the solar wind) are taken into account; see Leinert \& Grün (1990) and Liou \& Zook (1997). Liou \& Zook use the value $s w=0.35$, which is close to $\eta=1 / 3$, and the value $1 / 3$ also is mentioned in Holmes et al. (2003). However, the authors of the last two papers should use " $s w / Q_{\mathrm{pr}}$ " (our notification is " $\eta / \bar{Q}_{\mathrm{pr}}^{\prime}$ ") 
in their equation of motion - all terms with $\eta$, in our Eq. (4), contain the ratio $\eta / \bar{Q}_{\mathrm{pr}}^{\prime}$. The equation of motion for the effect of the solar wind (we neglect decreases of particle mass) is taken from Klačka (1994). We take into account that $\gamma_{\mathrm{T}} \equiv \sin \varepsilon$, where, according to Bruno et al. (2003), the angle $\varepsilon$ lies between 2.3 and 2.9 degrees for $u \approx 400 \mathrm{~km} \mathrm{~s}^{-1}$ and the value of $\varepsilon$ does not decrease with heliocentric distance. We will consider the value of $\varepsilon$ as a constant, independent of the heliocentric distance.

\section{Solar radiation and secular evolution of orbital elements}

Equation (4) describes the motion of spherical dust particles influenced by the effects of solar radiation - both electromagnetic and corpuscular (solar wind). Equation (4) can be used in perturbation equations of celestial mechanics, from which the secular evolution of orbital elements can be obtained for the case when the central acceleration is taken as $\mu(1-\beta) / r^{2}$. We do not take into account gravitational perturbation of the planet (subscript NG). Moreover, we assume that $\beta$ does not depend on the heliocentric distance (Kocifaj et al. 2006; Klačka et al. 2007). The secular evolution of the semimajor axis and eccentricity are:

$$
\begin{aligned}
\left\langle\frac{\mathrm{d} a}{\mathrm{~d} t}\right\rangle_{\mathrm{NG}}= & -\beta \frac{\mu}{c} \frac{2+3 e^{2}}{a\left(1-e^{2}\right)^{3 / 2}} \\
& \times\left\{1+\frac{\eta}{\bar{Q}_{\mathrm{pr}}^{\prime}}\left[1-\gamma_{\mathrm{T}} \frac{1}{2+3 e^{2}} \frac{1}{Z}\right]\right\}, \\
\left\langle\frac{\mathrm{d} e}{\mathrm{~d} t}\right\rangle_{\mathrm{NG}}= & -\beta \frac{\mu}{c} \frac{5 e / 2}{a^{2} \sqrt{1-e^{2}}} \\
& \times\left\{1+\frac{\eta}{\bar{Q}_{\mathrm{pr}}^{\prime}}\left[1-\gamma_{\mathrm{T}} \frac{1}{5} \frac{1-\sqrt{1-e^{2}}}{e^{2}} \frac{1}{Z}\right]\right\}, \\
Z \equiv & \sqrt{\frac{\mu(1-\beta)}{p}} \frac{1}{2 u}, p=a\left(1-e^{2}\right) .
\end{aligned}
$$

Equation (5) yields that the semimajor axis and eccentricity are decreasing functions of time for the case $\gamma_{\mathrm{T}} \equiv 0$. The particle approaches the Sun and sometimes may reach a suitable conditions for capture into a mean motion resonance. Being in the resonance, its secular evolution is characterized by a constant value of the semimajor axis defined by Eq. (2) or (3) (or, by the analogous equation for the interior resonance). Particles can be captured for thousands or even billions of years. This depends on the size of the particle (as $\beta \propto$ size $^{-1}$ ), the mass of the planet, type of resonance (the smaller the values of $p$ and $q$, the longer the capture times), and, also, on the initial orbital conditions at the instant of capture. Finally, when the capture ends, Eq. (5) correctly describes the behavior of the particle: the secular evolution of semimajor axis and eccentricity is, again, a decreasing function of time.

However, the real case $\gamma_{T} \neq 0$ also can yield a secular increase of the semimajor axis and eccentricity. Detailed numerical integrations show that the secular evolution of the semimajor axis can increase before the particle is captured into a mean motion orbital resonance with Neptune: see Sect. 6 for more details. This is the second case when such behavior was found, after the very complicated case of interaction between solar electromagnetic radiation and nonspherical dust particles (Klačka et al. 2005a,b).

\section{Secular evolution of eccentricity in a mean motion resonance}

The secular evolution of the semimajor axis is characterized by its constant value when the particle is in a resonance with a planet. To describe the secular evolution of the eccentricity of the particle in such a situation, we suppose that the planet is moving in a circular orbit around the Sun. In this case, we have the special gravitational problem of three bodies, and small nongravitational forces are also present. The gravitational problem is called the circular restricted three-body problem in celestial mechanics. At the end of the 19th century Tisserand found a quantity that does not change during the motion of the third body, whose mass is negligible in comparison to the masses of the planet and the Sun (see, e.g. Brouwer \& Clemence 1961). For our case, we can write the Tisserand parameter in the form

$C_{\mathrm{T}}=\frac{1-\beta}{a}+2 \sqrt{\frac{(1-\beta) a\left(1-e^{2}\right)}{a_{\mathrm{P}}^{3}}} \cos I$,

where $e$ is the eccentricity of the particle characterized by parameter $\beta$ and $I$ is the inclination of the particle's orbital plane to the plane of the planet's orbit. Tisserand's quantity $C_{\mathrm{T}}$ does not change only for the special case of the circular restricted problem of three bodies. However, Eq. (6) enables us to find the secular change of the eccentricity of the particle captured into a resonance. For this purpose, we will consider $I=0$ in Eq. (6). We can write

$\frac{\mathrm{d} C_{\mathrm{T}}}{\mathrm{d} t}=\frac{\partial C_{\mathrm{T}}}{\partial a}\left(\frac{\mathrm{d} a}{\mathrm{~d} t}\right)_{\text {total }}+\frac{\partial C_{\mathrm{T}}}{\partial e}\left(\frac{\mathrm{d} e}{\mathrm{~d} t}\right)_{\text {total }}$,

for the total time derivative of the Tisserand quantity $C_{\mathrm{T}}$ defined by Eq. (6). However, according to Eq. (4), time derivatives of the semimajor axis and eccentricity of the particle are caused by gravitational perturbations of the planet (these terms will be denoted by the subscript $\mathrm{G}$ ) and nongravitational perturbations caused by solar electromagnetic radiation and the solar wind (these terms will be denoted by the subscript NG):

$$
\begin{aligned}
& \left(\frac{\mathrm{d} a}{\mathrm{~d} t}\right)_{\text {total }}=\left(\frac{\mathrm{d} a}{\mathrm{~d} t}\right)_{\mathrm{G}}+\left(\frac{\mathrm{d} a}{\mathrm{~d} t}\right)_{\mathrm{NG}}, \\
& \left(\frac{\mathrm{d} e}{\mathrm{~d} t}\right)_{\text {total }}=\left(\frac{\mathrm{d} e}{\mathrm{~d} t}\right)_{\mathrm{G}}+\left(\frac{\mathrm{d} e}{\mathrm{~d} t}\right)_{\mathrm{NG}} .
\end{aligned}
$$

On the basis of Eqs. (7), (8) we can write

$$
\begin{aligned}
\frac{\mathrm{d} C_{\mathrm{T}}}{\mathrm{d} t}= & \frac{\partial C_{\mathrm{T}}}{\partial a}\left\{\left(\frac{\mathrm{d} a}{\mathrm{~d} t}\right)_{\mathrm{G}}+\left(\frac{\mathrm{d} a}{\mathrm{~d} t}\right)_{\mathrm{NG}}\right\} \\
& +\frac{\partial C_{\mathrm{T}}}{\partial e}\left\{\left(\frac{\mathrm{d} e}{\mathrm{~d} t}\right)_{\mathrm{G}}+\left(\frac{\mathrm{d} e}{\mathrm{~d} t}\right)_{\mathrm{NG}}\right\} .
\end{aligned}
$$

According to Tisserand, gravitational terms alone do not change the value of $C_{\mathrm{T}}$ :

$\frac{\partial C_{\mathrm{T}}}{\partial a}\left(\frac{\mathrm{d} a}{\mathrm{~d} t}\right)_{\mathrm{G}}+\frac{\partial C_{\mathrm{T}}}{\partial e}\left(\frac{\mathrm{d} e}{\mathrm{~d} t}\right)_{\mathrm{G}}=0$.

Putting Eq. (10) into Eq. (9):

$\frac{\mathrm{d} C_{\mathrm{T}}}{\mathrm{d} t}=\frac{\partial C_{\mathrm{T}}}{\partial a}\left(\frac{\mathrm{d} a}{\mathrm{~d} t}\right)_{\mathrm{NG}}+\frac{\partial C_{\mathrm{T}}}{\partial e}\left(\frac{\mathrm{d} e}{\mathrm{~d} t}\right)_{\mathrm{NG}}$.

If we are interested in secular changes of orbital elements $a$ and $e$, then the particle's stay in the resonance is characterized by the 
relation $\langle\mathrm{d} a / \mathrm{d} t\rangle=0$ : for a function $a$ of the property $a(T)=a(0)$ the relation $\langle\mathrm{d} a / \mathrm{d} t\rangle=(1 / T) \int_{0}^{T}(\mathrm{~d} a / \mathrm{d} t) d t=(a(T)-a(0)) / T=0$ holds. After averaging over the period of the resonant libration of the semimajor axis, Eq. (7) reduces to

$$
\left\langle\frac{\mathrm{d} C_{\mathrm{T}}}{\mathrm{d} t}\right\rangle=\frac{\partial C_{\mathrm{T}}}{\partial e}\left\langle\frac{\mathrm{d} e}{\mathrm{~d} t}\right\rangle .
$$

From averaging of Eq. (11) we have

$$
\left\langle\frac{\mathrm{d} C_{\mathrm{T}}}{\mathrm{d} t}\right\rangle=\frac{\partial C_{\mathrm{T}}}{\partial a}\left\langle\frac{\mathrm{d} a}{\mathrm{~d} t}\right\rangle_{\mathrm{NG}}+\frac{\partial C_{\mathrm{T}}}{\partial e}\left\langle\frac{\mathrm{d} e}{\mathrm{~d} t}\right\rangle_{\mathrm{NG}} .
$$

Equations (12) and (13) yield

$$
\left\langle\frac{\mathrm{d} e}{\mathrm{~d} t}\right\rangle=\left\langle\frac{\mathrm{d} e}{\mathrm{~d} t}\right\rangle_{\mathrm{NG}}+\frac{\partial C_{\mathrm{T}} / \partial a}{\partial C_{\mathrm{T}} / \partial e}\left\langle\frac{\mathrm{d} a}{\mathrm{~d} t}\right\rangle_{\mathrm{NG}},
$$

for the total secular change of the eccentricity of the particle. Equations (5), (6) and (14) finally yield

$$
\begin{aligned}
\left\langle\frac{\mathrm{d} e}{\mathrm{~d} t}\right\rangle= & \beta \frac{\mu}{c} \frac{\left(1-e^{2}\right)^{1 / 2}}{a^{2} e}(X-Y), \\
X= & \left(1+\frac{\eta}{\bar{Q}_{\mathrm{pr}}^{\prime}}\right)\left\{1-\frac{\left(1+3 e^{2} / 2\right) \sqrt{1-\beta}}{\left(a / a_{\mathrm{P}}\right)^{3 / 2}\left(1-e^{2}\right)^{3 / 2}}\right\}, \\
Y= & \gamma_{\mathrm{T}} \frac{\eta}{\bar{Q}_{\mathrm{pr}}^{\prime}} \frac{u}{\sqrt{\mu(1-\beta) / a}} \\
& \times\left\{1-\frac{\sqrt{1-\beta}}{\left(a / a_{\mathrm{P}}\right)^{3 / 2}\left(1-e^{2}\right)}\right\},
\end{aligned}
$$

where the ratio $a / a_{\mathrm{P}}$ can be expressed through the ratio $n_{\mathrm{P}} / n$ from Eq. (2). The obtained equation reduces to the equation of Liou \& Zook (1997) under the assumption that $\eta \equiv 0$. The special case $\gamma_{\mathrm{T}} \equiv 0$ reduces Eq. (15) to the equation consistent with Eq. (83) in Klačka \& Kocifaj (2006a; see also Eq. (20) in Klačka $\&$ Kocifaj 2006b). Equation (15) was obtained for the circular restricted three-body problem with the action of nongravitational forces. We can apply it to planets with almost circular orbits, as an approximation to reality. We will use Neptune in an orbit of eccentricity 0.011 , which is close to a circular orbit.

Equation (15) determines the secular evolution of eccentricity of the particle characterized by the values $\beta$ and $\bar{Q}_{\mathrm{pr}}^{\prime}$, if the particle is captured into a mean motion resonance. This equation enables us to determine its detailed evolution. However, important qualitative behavior can be found very easily for the case $\gamma_{\mathrm{T}} \equiv 0$. If we take some special mean motion resonance, we already know the value $n / n_{\mathrm{P}}$. If the initial (secular) eccentricity is small enough, then $\langle\mathrm{d} e / \mathrm{d} t\rangle$ is always positive and the eccentricity of the particle is an increasing function of time during the stay of the particle in the exterior mean motion orbital resonance. The eccentricity of the particle can only asymptotically approach the limiting value $e_{\mathrm{lim}}$ given by the condition that the term in the combined brackets is zero: $n_{\mathrm{P}} / n=\left(1+3 e_{\lim }^{2} / 2\right) /\left(1-e_{\lim }^{2}\right)^{3 / 2}$ (the factor $\left(1+m_{\mathrm{P}} / M_{\odot}\right)^{1 / 2}$ is for the Solar system planets approximately equal to 1 so we can neglect it). If the initial eccentricity is large enough, then $\langle\mathrm{d} e / \mathrm{d} t\rangle$ is always negative and the eccentricity of the particle is a decreasing function of time, during the stay of the particle in the exterior mean motion resonance. The eccentricity of the particle can only asymptotically approach the limiting value $e_{\mathrm{lim}}$. If one considers interior mean motion orbital resonances, then the secular eccentricity of the particle is always a decreasing function of time.
Table 1. Values of $\varepsilon_{\text {crit }}$ (in degrees) for various resonances with Neptune in a circular orbit.

\begin{tabular}{ccc}
\hline \hline res & $\begin{array}{c}\varepsilon_{\text {crit }} \\
Q_{\mathrm{pr}}^{\prime}=1 / 2\end{array}$ & $\begin{array}{c}\varepsilon_{\text {crit }}^{\prime} \\
Q_{\mathrm{pr}}=1\end{array}$ \\
\hline $2: 1$ & 1.542 & 2.469 \\
$3: 2$ & 1.696 & 2.717 \\
$4: 3$ & 1.764 & 2.826 \\
$5: 4$ & 1.802 & 2.887 \\
$6: 5$ & 1.827 & 2.926 \\
$1: 2$ & 2.444 & 3.918 \\
$2: 3$ & 2.221 & 3.559 \\
$3: 4$ & 2.136 & 3.423 \\
$4: 5$ & 2.090 & 3.350 \\
$5: 6$ & 2.062 & 3.305 \\
\hline
\end{tabular}

The crucial question emerges: are the features, presented above, typical features of real dust particles and real physical forces? We know, on the basis of several papers on the interaction between nonspherical dust grains and solar electromagnetic radiation, that the above discussed behavior may not hold. Our consideration of the transversal component of the solar wind velocity shows that a similar situation may hold not only for nonspherical dust grains, but also for spherical grains. More realistic properties of dust grains and/or physical forces acting on the grains show that there is no real physical argument for the existence of the limiting value(s) $e_{\mathrm{lim}}$, in general.

Let us consider real nonzero values of $\varepsilon$, or, $\gamma_{\mathrm{T}} \equiv \sin \varepsilon$. Eq. (15) yields that there may exist a critical value of $\varepsilon_{\text {crit }}$ for the mean motion orbital resonances. For values $\varepsilon<\varepsilon_{\text {crit }}$ secular evolution of eccentricity qualitatively differs from the case $\varepsilon>\varepsilon_{\text {crit }}$. The case $\varepsilon<\varepsilon_{\text {crit }}$ yields, qualitatively, the same secular evolution of eccentricity as the case $\varepsilon=0$. However, the case $\varepsilon>\varepsilon_{\text {crit }}$ yields that eccentricity is a decreasing function of time for the exterior mean motion orbital resonances and eccentricity exhibits two monotonous parts (an increasing and a decreasing one) with an asymptotic behavior for the interior resonances. For a given type of resonance, planet and $\eta / \bar{Q}_{\mathrm{pr}}^{\prime}$, the value of $\varepsilon_{\text {crit }}$ is practically independent of the values $\beta$, if $\beta$ is within the interval $\beta \in\langle 0.001,0.1\rangle$ - the decrease of $\varepsilon_{\text {crit }}$ with an increasing $\beta$ is characterized by the condition of the relative change $1-\varepsilon_{\text {crit }}(\beta=0.001) / \varepsilon_{\text {crit }}(\beta=0.1) \approx 0.03-0.04$. The value of $\varepsilon_{\text {crit }}$ is a decreasing function of $\eta / \bar{Q}_{\mathrm{pr}}^{\prime}$, for both exterior and interior resonances. The value of $\varepsilon_{\text {crit }}$ is: i) an increasing function of $p$ for the first order exterior resonances $(p+1) / p$, and; ii) a decreasing function of the order of the resonances $q$. For the case of the exterior mean motion orbital resonances with planet Neptune (we use $a_{\mathrm{P}}=30 \mathrm{AU}$ ), and $\beta=0.01$, one obtains that $\varepsilon_{\text {crit }}<2.9^{\circ}$ holds only for the case $\eta / \bar{Q}_{\mathrm{pr}}^{\prime}>0.27044$, or $\bar{Q}_{\mathrm{pr}}^{\prime}<1.23256$. The value of $\varepsilon_{\text {crit }}$ is: i) a decreasing function of $p$ for the first order interior resonances $p /(p+1)$, and; ii) an increasing function of the order of the interior resonances $q$. For the case of the interior mean motion orbital resonances with Neptune, and $\beta=0.01$, one obtains that $\varepsilon_{\text {crit }}<2.9^{\circ}$ holds only for the case $\eta / \bar{Q}_{\mathrm{pr}}^{\prime}>0.51$, or $\bar{Q}_{\mathrm{pr}}^{\prime}<0.6536$.

Table 1 presents values of $\varepsilon_{\text {crit }}$ for $\beta=0.01, \eta=1 / 3$, $\bar{Q}_{\mathrm{pr}}^{\prime}=1 / 2$ and 1 , for several exterior and interior resonances with Neptune.

Figures 1 and 2 depict secular evolution of the particle's eccentricity in the mean motion 2:1 orbital resonance with Neptune for the case $\beta=0.01$ and $\eta / \bar{Q}_{\mathrm{pr}}^{\prime}=2 / 3$. Figure 1 holds for $\varepsilon=0^{\circ}$ and Fig. 2 holds for $\varepsilon=2.5^{\circ}$. If $\varepsilon<\varepsilon_{\text {crit }}=1.542^{\circ}$, then the 


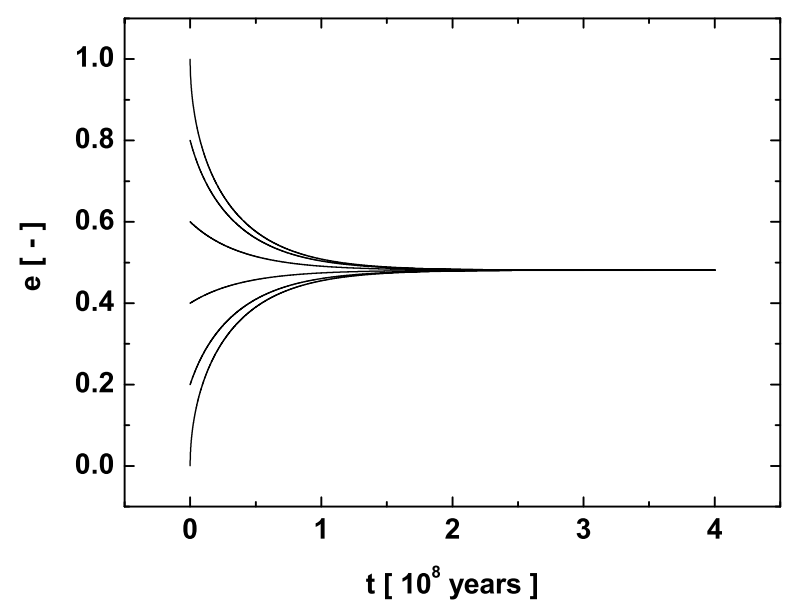

Fig. 1. Evolution of eccentricity obtained from Eq. (15). A dust particle with $\beta=0.01$ and $\eta / \bar{Q}_{\mathrm{pr}}^{\prime}=2 / 3$ is captured in an exterior mean motion 2:1 orbital resonance with Neptune. The solar wind parameter $\varepsilon=0$. Evolution curves for various initial conditions of eccentricity are shown.

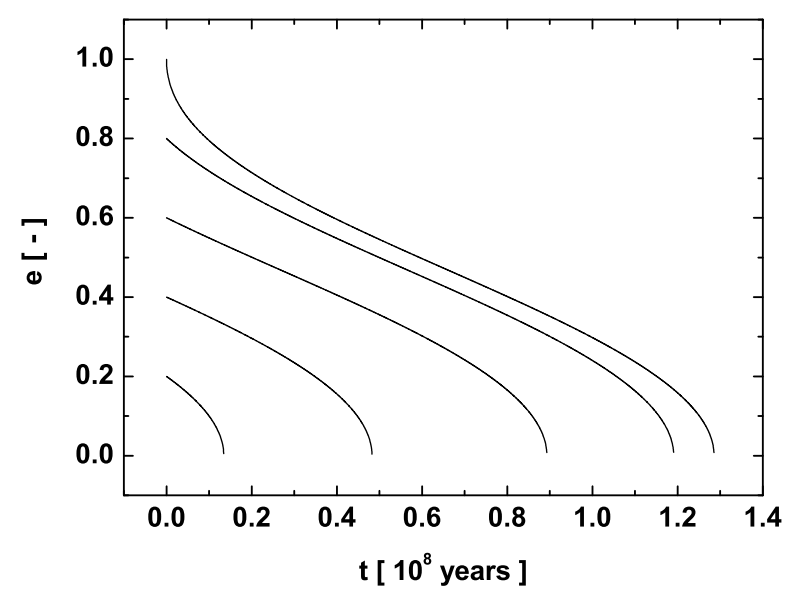

Fig. 2. Evolution of eccentricity obtained from Eq. (15). A dust particle with $\beta=0.01$ and $\eta / \bar{Q}_{\mathrm{pr}}^{\prime}=2 / 3$ is captured in an exterior mean motion 2:1 orbital resonance with Neptune. Solar wind parameter $\varepsilon=2.5^{\circ}$. Evolution curves for various initial conditions are shown.

behavior is similar to Fig. 1 . If $\varepsilon>1.542^{\circ}$, then the secular evolution of eccentricity is similar to Fig. 2 . The detailed numerical integrations of Eq. (4) are consistent with Eq. (15).

\section{Secular evolution of orbital elements near resonances}

Figure 3 presents the orbital evolution of dust grains obtained by numerical integration of Eq. (4) with $\beta=0.01$ for $\varepsilon=2.5^{\circ}$ and $\eta / \bar{Q}_{\mathrm{pr}}^{\prime}=2 / 3$.

Secular evolution of the semimajor axis is an increasing function of time before capture into the 20:9 resonance with Neptune. The same holds after ejection of the particle from the resonance. The cases when $\langle\mathrm{d} a / \mathrm{d} t\rangle>0$ can be found from Eq. (5) for various combinations of $\eta / \bar{Q}_{\mathrm{pr}}^{\prime}, \beta, a$ and $e-$ this relation shows when $\langle\mathrm{d} a / \mathrm{d} t\rangle>0$ before the grain is captured into an exterior/interior resonance. Up to now, only nonspherical dust grains were captured when the secular change of semimajor axis was an increasing function of time near the zone of the resonance (Klačka et al. 2005a,b; Klačka \& Kocifaj 2006a,b).

Secular evolution of eccentricity is a decreasing function of time $\left(\varepsilon>\varepsilon_{\text {crit }}=1.488^{\circ}\right)$ : the initial resonance value $e_{\text {initial }}=0.3$
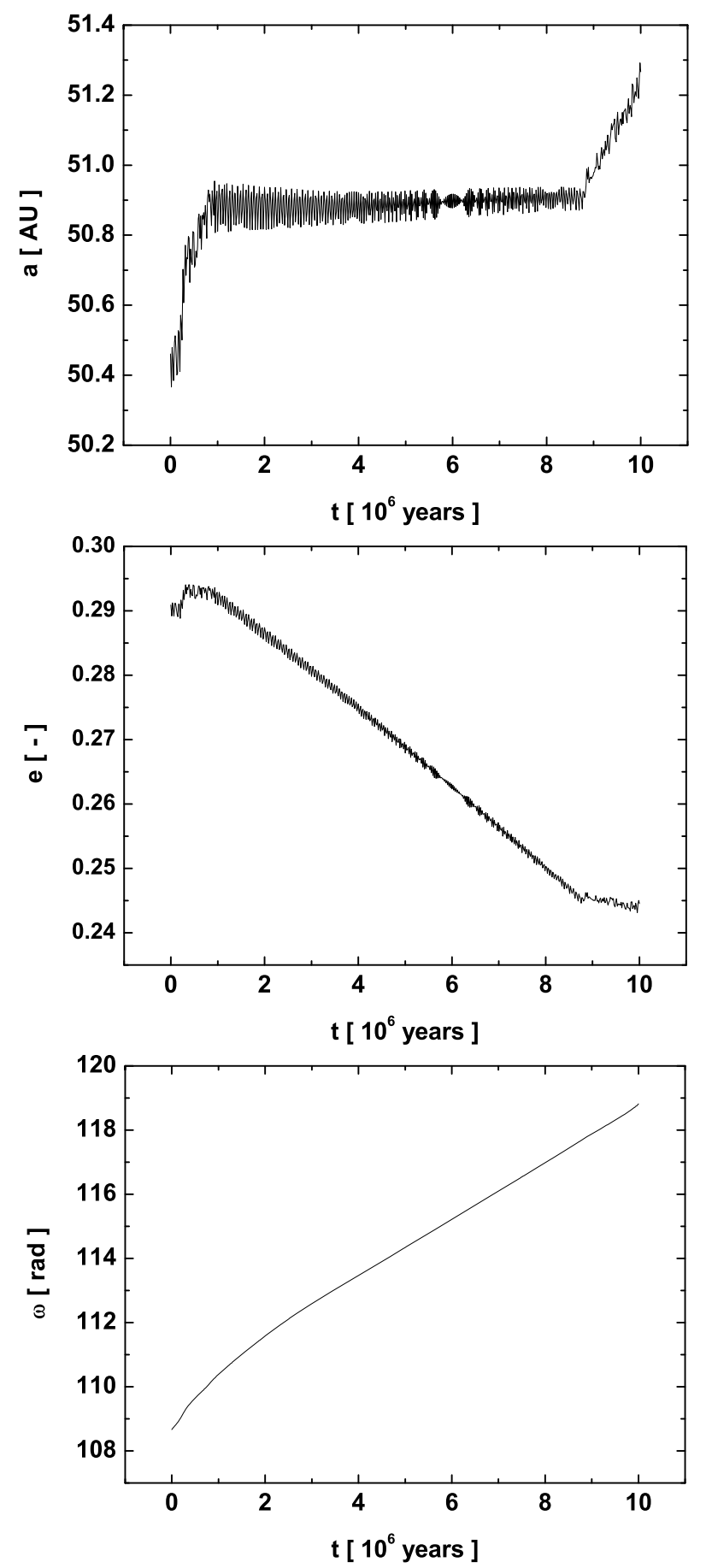

Fig. 3. Orbital evolution of spherical dust particles with $\beta=0.01$ and $\eta / \bar{Q}_{\mathrm{pr}}^{\prime}=2 / 3$ captured in the exterior mean motion 20:9 resonance with planet Neptune: $a$ - semimajor axis, $e$ - eccentricity, $\omega$-argument of perihelion.

is less than the limiting value $e_{\lim } \approx 0.5154$ for the case $\varepsilon=$ $\gamma_{\mathrm{T}}=0$ [see Eq. (15): $n_{\mathrm{P}} / n=\left(1+3 e_{\lim }^{2} / 2\right) /\left(1-e_{\mathrm{lim}}^{2}\right)^{3 / 2}$ ] and $\langle\mathrm{d} e / \mathrm{d} t\rangle\langle 0$. The numerical secular evolution of eccentricity is consistent with Eq. (15). The fact that no limiting value of eccentricity exists in the resonance was obtained not only for nonspherical grains, but also for spherical grains in the elliptical restricted three-body problem with the action of electromagnetic radiation of the central star (Pástor et al. 2007). We have just shown that the discussed cases also can occur for spherical dust grains, if a more realistic disturbing force is taken into account together with the circular restricted three-body problem. 
As for the shift of perihelion (secular evolution of the argument of perihelion), a $\langle\mathrm{d} \omega / \mathrm{d} t\rangle>0$ and capture into the resonance does not significantly influence its behavior. Numerical calculations yield that $\langle\mathrm{d} \omega / \mathrm{d} t\rangle>0$ and $\partial\langle\mathrm{d} \omega / \mathrm{d} t\rangle_{\text {res }} / \partial \varepsilon<0$. This can be easily understood in terms of the shift of perihelion given by Eq. (4) for the central acceleration $-\mu(1-\beta) \boldsymbol{e}_{\mathrm{R}} / r^{2}$ and neglecting the gravitational perturbation of the planet:

$$
\begin{aligned}
\left\langle\frac{\mathrm{d} \omega}{\mathrm{d} t}\right\rangle_{\mathrm{NG}}= & -\beta \frac{\eta}{\bar{Q}_{\mathrm{pr}}^{\prime}} \frac{\mu}{c} \frac{1}{a^{2} \sqrt{1-e^{2}}} \\
& \times\left\{\gamma_{\mathrm{T}} \frac{1-\sqrt{1-e^{2}}}{e^{2}}-Z\right\},
\end{aligned}
$$

where $Z$ is defined in Eq. (5).

Figure 4 presents the orbital evolution of dust grains with $\beta=0.01$ for $\varepsilon=2.5^{\circ}$ and $\eta / \bar{Q}_{\mathrm{pr}}^{\prime}=2 / 3$. The Secular evolution of the semimajor axis is an increasing function of time before the grain is captured into the interior mean motion orbital 15:17 resonance with Neptune. The value $\varepsilon_{\text {crit }}$ for this resonance is $2.024^{\circ}$, i.e. $\varepsilon>\varepsilon_{\text {crit }}$ and due to the results obtained in Sect. 5, the eccentricity is an increasing function of time and asymptotically approaches the limiting value $e_{\text {lim }}=0.124$. This is consistent with numerical simulations. As for the shift of perihelion, $\langle\mathrm{d} \omega / \mathrm{d} t\rangle>0$ before the capture into the resonance and $\langle\mathrm{d} \omega / \mathrm{d} t\rangle$ is a discontinuous function of time at the moment of capture this is the difference to the exterior orbital resonance (compare with Fig. 3). $\langle\mathrm{d} \omega / \mathrm{d} t\rangle>0$ before the capture - this can be understood in terms of the shift of perihelion given by Eq. (4) for the central acceleration $-\mu(1-\beta) \boldsymbol{e}_{\mathrm{R}} / r^{2}$ and neglecting gravitational perturbation of the planet, see also Eq. (16).

\section{Conclusion}

We investigated the action of solar electromagnetic radiation and solar wind on dynamics of spherical interplanetary dust particles. Concentration was applied to important properties of motion of dust grains in the zone of mean motion orbital resonances with Neptune moving in a circular orbit (the real eccentricity of Neptune is so small that the approximation by a circular orbit yields correct results for the considered gravitational and nongravitational forces).

Extrapolating of the observed non-radial direction of the solar wind, we have found that spherical dust grains also can be captured in the resonances even in the case when secular change of the semimajor axis is an increasing function of time near the zone of the resonances. Resonant trapping is not normally expected for diverging orbits such as the trapping presented in Figs. 3 and 4; the only case known up to now has been the capture of nonspherical dust grains in the resonances. We have shown that a new physical force acting on spherical dust grains can generate the same qualitative result. This kind of force may act also in the vicinity of other stars in the form of the stellar wind.

We have succeeded in determining the secular evolution of eccentricity of a spherical particle in a mean motion orbital resonance in an analytical way. The obtained result is represented by Eq. (15). Equation (15) is a (corrected) generalization of the results obtained by Liou \& Zook (1997) \& Klačka \& Kocifaj $(2006 a, b)$. The detailed numerical calculations of Eq. (4) are consistent with Eq. (15).

The non-radial component of the solar wind used here has an important consequence not only for the capture of the particle into the resonance. Spherical interplanetary dust grains orbiting
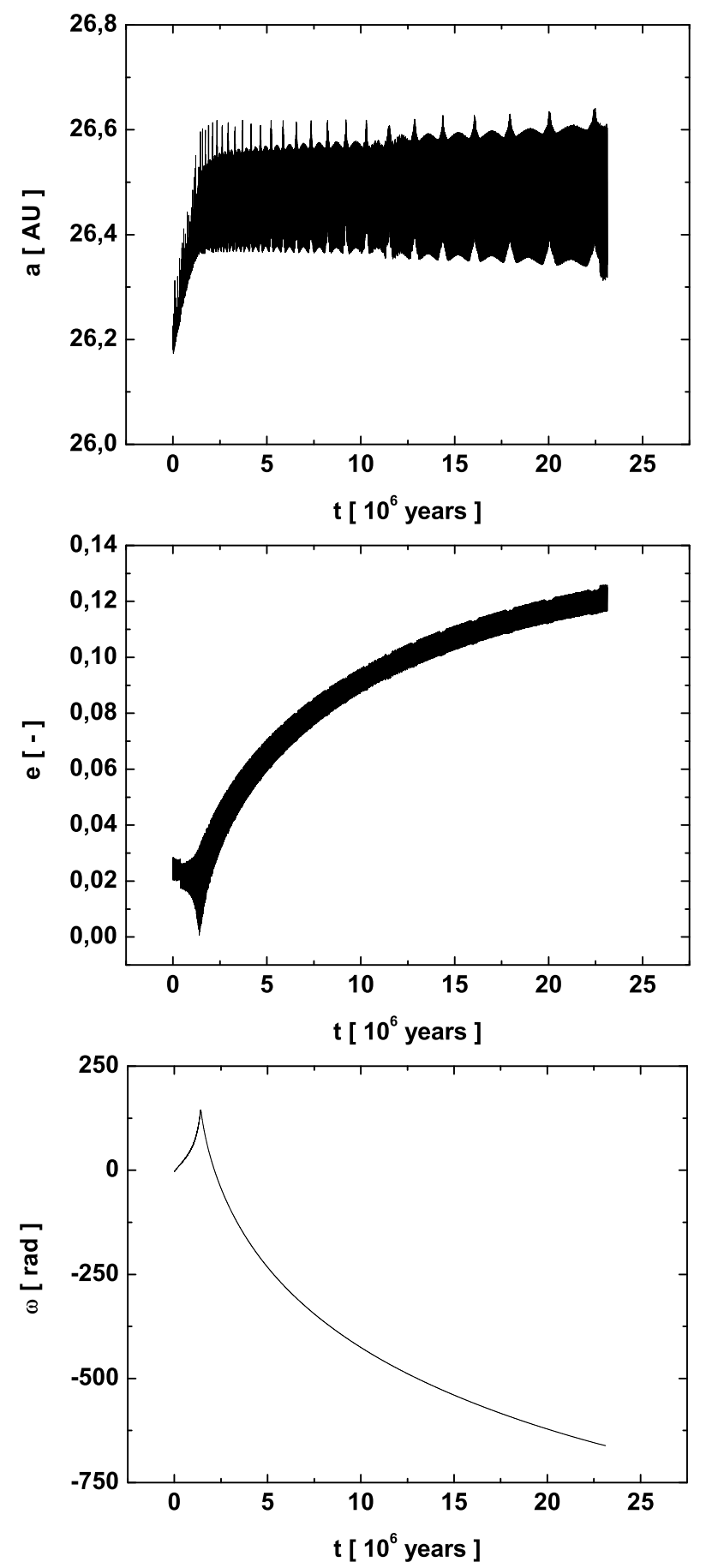

Fig. 4. Orbital evolution of spherical dust particles with $\beta=0.01$ and $\eta / \bar{Q}_{\mathrm{pr}}^{\prime}=2 / 3$ captured in the interior mean motion orbital 15:17 resonance with Neptune.

the Sun at distances comparable to the heliocentric distance of Neptune, or even larger, may exhibit a secular increase of their semimajor axis. This is a new result, since only the effects of solar electromagnetic radiation and the radial component of the solar wind have been considered up to now and both of them lead to a secular decrease of the semimajor axis for spherical dust grains. However, this does not mean that dust particles do not spiral toward the sun. Real dust grains are arbitrarily shaped and interaction of electromagnetic radiation with nonspherical dust particles may be more important than the effect of the non-radial component of the solar wind. This holds for particles not larger 
than several microns (Klačka \& Kocifaj 2002): if we consider particles of several tens of microns in size, then the effect of electromagnetic radiation cannot produce divergent orbits, while the non-radial component of the solar wind can generate such a behavior. The effect of a non-radial component of the solar wind velocity depends, in reality, on the physical properties of the particle, optical properties (the ratio $\eta / \bar{Q}_{\mathrm{pr}}^{\prime}$ in Eqs. (5)) and orbital elements (eccentricity $e$ and parameter $p$ are present in chain brackets in the first of Eqs. (5)), and some of their combinations produce divergent orbits, others produce secular decreases of the semimajor axis.

If the dust rings in mean motion orbital resonances with Neptune are observed, then the captured grains have to be not only from the outer part of the Solar System, but also from the zone situated inside Neptune's orbit. This is a consequence not only of the action of the non-radial component of the solar wind, but also of the action of solar electromagnetic radiation on nonspherical dust particles. This differs from the action of the P-R effect and the radial solar wind. Comparison of capture times found in our paper with those obtained for nonspherical particles suggests that the solar wind effect is less significant than the effect of solar electromagnetic radiation on nonspherical grains with larger values of the impact ratio (if the ratio between the largest and the smallest size of the particle is larger than, say, 1.3). These considerations hold for micron-sized particles. If the particle sizes are of several tens of microns, then the non-radial component of the solar wind velocity plays a more important role than the effect of the nonsphericity of the particle interacting with the solar electromagnetic radiation.

Acknowledgements. The authors thank to Dr. A. Breen for discussions on the non-radial component of solar wind. The paper was supported by the Scientific Grant Agency VEGA (grant No. 1/3074/06) and by the Comenius University grants $U K / 371 / 2007$ and $U K / 379 / 2007$.

\section{References}

Beaugé, C., \& Ferraz-Mello, S. 1994, Icarus, 110, 239

Brouwer, D., \& Clemence, G. M. 1961, Methods of Celestial Mechanics (New York: Academic Press)

Brownlee, D. E. 1994, Nature, 369, 706
Bruno, R., Carbone, V., Sorriso-Valvo, L., \& Bavassano, B. 2003, J. Geophys. Res., 108(A3), 1130

Dermott, S. F., Jayaraman, S., Xu, Y. L., Gustafson, B. A. S., \& Liou, J. C. 1994, Nature, 369, 719

Dohnanyi, J. S. 1978, Particle dynamics in Cosmic Dust, ed. J. A. M. McDonnell (Chichester: Wiley), 527

Grün, E., Gustafson, B., Mann, I., et al. 1994, A\&A, 286, 915

Holmes, E. K., Dermott, S. F., Gustafson, B. A. S., \& Grogan, K. 2003, ApJ, 597,1211

van de Hulst, H. C. 1981, Light Scattering by Small Particles (New York: Dover Publications)

Hundhausen, A. J. 1972, Coronal Expansion and Solar Wind (Berlin: SpringerVerlag)

Jackson, A. A., \& Zook, H. A. 1989, Nature, 337, 629

Kehoe, T. J. J., Dermott, S. F., \& Grogan, K. 2002, Mem. S. A. It., 73, 684

Klačka, J. 1994, Earth Moon Planets, 64, 125

Klačka, J. 2000, [arXiv: astro-ph/0008510]

Klačka, J. 2004, Cel. Mech. and Dynam. Astron., 89, 1

Klačka, J. 2008, [arXiv: 0807.2795]

Klačka, J., \& Kocifaj, M. 2002, On applicability of Poynting-Robertson effect, in Proceedings of Asteroids, Comets, Meteors ACM 2002, ed. B. Warmbein (NoordwijkESA: Publications Division), 169

Klačka, J., \& Kocifaj, M. 2006a, Effect of electromagnetic radiation on dynamics of cosmic dust particles, in Space Science: New Research, ed. Nick S. Maravell (New York: Nova Science Publishers), 245

Klačka, J., \& Kocifaj, M. 2006b, J. Quant. Spectrosc. Radiat. Transfer, 100, 187 Klačka, J., Kocifaj, M., \& Pástor, P. 2005a, J. Phys.: Conf. Ser., 6, 126

Klačka, J., Kocifaj, M., \& Pástor, P. 2005b, Effect of radiation on nonspherical particles in resonances with large planets, in 8th Conference on Electromagnetic and Light Scattering by Nonspherical Particles: Theory, Measurements and Applications, ed. F. Moreno, J. J. López-Moreno, O. Munoz, \& A. Molina (Instituto de Astrofisica de Granada), 156

Klačka, J., Kocifaj, M., Pástor, P., \& Petržala, J. 2007, A\&A, 464, 127

Kocifaj, M., Klačka, J., \& Horvath, H. 2006, MNRAS, 370, 1876

Leinert, C., \& Grün, E. 1990, Interplanetary dust, in Physics of the Inner Heliosphere I, ed. R. Schwenn, E. Marsch (Berlin: Springer-Verlag), 207

Liou, J.-Ch., \& Zook, H. A. 1995, Icarus, 113, 403

Liou, J.-Ch., \& Zook, H. A. 1997, Icarus, 128, 354

Liou, J.-Ch., Zook, H. A., \& Jackson, A. A. 1995, Icarus, 116, 186

Liou, J.-Ch., Zook, H. A., \& Jackson, A. A. 1999, Icarus, 141, 13

Marzari, F., \& Vanzani, V. 1994, A\&A, 283, 275

Pástor, P., Klačka, J., \& Kómár, L. 2007, Cel. Mech. Dynam. Astron, submitted, [arXiv:0807.1425]

Reach, W. T., Franz, B. A., Welland, J. L., et al. 1995, Nature, 374, 521

Robertson, H. P. 1937, MNRAS, 97, 423

Stix, M. 2002, The Sun (Berlin: Springer-Verlag)

Šidlichovský, M., \& Nesvorný, D. 1994, A\&A, 289, 972

Wisdom, J. 1980, AJ, 85, 1122 